\title{
A QUASI-STATIC METHOD FOR DETERMINING THE CHARACTERISTICS OF A MOTION CAPTURE CAMERA SYSTEM IN A "SPLIT-VOLUME" CONFIGURATION
}

\author{
Chris Miller ${ }^{1}$, Ajitkumar Mulavara ${ }^{1}$, and Jacob Bloomberg ${ }^{2}$ \\ ${ }^{1}$ Neurosciences Laboratory, Wyle Laboratories, Houston, TX, USA \\ ${ }^{2}$ Neurosciences Laboratory, Johnson Space Center, Houston, TX, USA \\ E-mail: chris.miller1@jsc.nasa.gov
}

\section{INTRODUCTION}

To confidently report any data collected from a video-based motion capture system, its functional characteristics must be determined, namely accuracy, repeatability and resolution. Many researchers (Haggard and Wing, 1990; Klein and DeHaven, 1995; Thornton, et al., 1998; VanderLinden, et al., 1992) have examined these characteristics with motion capture systems, but they used only two cameras, positioned 90 degrees to each other. Everaert, et al. (1999) used 4 cameras, but all were aligned along major axes (two in $\mathrm{x}$, one in $\mathrm{y}$ and $\mathrm{z}$ ). Richards (1998) compared the characteristics of different commercially available systems set-up in practical configurations, but all cameras viewed a single calibration volume. The purpose of this study was to determine the accuracy, repeatability and resolution of a 6-camera Motion Analysis system in a split-volume configuration using a quasistatic methodology.

\section{METHODS}

Per our standard data collection protocol for treadmill walking, six Hi-Res Falcon cameras (Motion Analysis, Santa Rosa, CA) were setup in a split-volume configuration to enable whole-body kinematic measurements in a confined lab space. Three cameras were aimed to capture lower limb motion (i.e., waist-down); three cameras were aimed to capture upper body motion (i.e., waist-up).
Each camera was positioned between 1.8 and 2.5 meters from the center of the calibration volume (size $0.75 \times 0.50 \times 1.77$ meters), in a distribution that covered from "two o'clock" to "seven o'clock" in the xyplane (with 12 o'clock being in the direction of progression).

A retroreflective marker ( $25 \mathrm{~mm}$ dia.) was fixed to a linearly translating table. The table was moved via a screw micrometer with a resolution of 0.001 inches $(0.0254$ $\mathrm{mm})$. Retroreflective tape was wrapped around the end of the micrometer handle, such that the tape was hidden while the operator turned the handle. Data were collected on the marker's position as the table was moved from 0.000 to 1.000 inches ( 0 to $25.4 \mathrm{~mm}$ ) by increments of $0.001 \mathrm{inch}$. Each incremental (i.e., quasi-static) position was held for 2 seconds with the operator's hand away from the micrometer handle (i.e., not covering the handle tape).

The table was placed at each of five different heights, centered in the xy-plane of the calibration volume. The five heights corresponded to the following regions: headlevel (upper); chest (mid-upper); waist (middle); mid-thigh (mid-lower), and shank (lower). Two data files for each region were collected on separate days.

The handle tape, when visible, was tracked as an indicator. A window of 31 frames was defined within each quasi-static position 
(i.e., when the handle marker was visible), centered about its mid-frame point. Within each window, the mean and standard deviation (SD) of the displacements from the initial position were computed. Calculated displacements were subtracted from actual displacements (as measured by the micrometer position) to compute error.

To determine resolution, the mean displacements at each quasi-static position were analyzed with an ANOVA $(p<0.01)$ with a Tukey post-hoc test for significance. Differences between consecutive significantly different displacements were calculated, and the mean and SD of the differences were computed for each region. To determine the accuracy, the absolute values of the mean errors of the marker displacement for each position were calculated, and the mean and SD for each region were computed. Repeatability was determined by averaging the SDs for each position, and $99 \%$ confidence limits $\left(3 * \mathrm{SD}_{\mathrm{avg}}\right)$ were calculated for each region.

\section{RESULTS AND DISCUSSION}

The results are shown in Table 1. It should be noted that with the split-volume camera setup, the characteristic values were consistent across all regions. The actual resolution calculated was $0.06 \mathrm{~mm}$, but this was rounded up to $0.1 \mathrm{~mm}$ for practical reporting. This resolution is approximately
$0.40 \%$ of the marker size $(25 \mathrm{~mm})$ and $0.02 \%$ of the shortest calibration volume dimension $(502 \mathrm{~mm})$. Repeatability also was computed to $0.1 \mathrm{~mm}$, based on the $99 \%$ confidence limit definition. Regional means for accuracy ranged from $0.05 \mathrm{~mm}$ to $0.16 \mathrm{~mm}$.

\section{SUMMARY}

The quasi-static method is an effective method for determining the characteristics of a motion capture system in a split-volume configuration. Values were consistent across all regions of the calibration volume. This method permits precise measurement over the entire body during treadmill locomotion within a confined lab space.

\section{REFERENCES}

Everaert, D.G., et al. (1999). Arch Phys Med Rehabilitation, 80, 1082-9.

Haggard, P., Wing, A.M. (1990). J. Motor Behavior, 22, 315-21.

Klein, P.J., DeHaven, J.J. (1995). Arch Phys Med Rehabilitation, 76, 183-9.

Richards, J.G. (1998). Proceedings of $5^{\text {th }}$ International Symposium on the 3-D Analysis of Human Movement, 1-9. Thornton, M.J., et al. (1998). Clinical Biomechanics, 13, 452-4.

Vander Linden, D.W., et al. (1992). Physical Therapy, 72, 300-5.

Table 1: Resolution, accuracy (mean \pm SD) and repeatability (99\% CL) statistics (in mm).

\begin{tabular}{|c|c|c|c|c|c|c|}
\cline { 2 - 7 } \multicolumn{1}{c|}{} & \multicolumn{2}{c|}{ Resolution } & \multicolumn{2}{c|}{ Accuracy } & \multicolumn{2}{c|}{ Repeatability } \\
\hline Region & Trial 1 & Trial 2 & Trial 1 & Trial 2 & Trial 1 & Trial 2 \\
\hline Upper & $0.059 \pm 0.020$ & $0.062 \pm 0.020$ & $0.097 \pm 0.078$ & $0.103 \pm 0.083$ & 0.102 & 0.102 \\
\hline Mid-upper & $0.055 \pm 0.019$ & $0.064 \pm 0.014$ & $0.054 \pm 0.041$ & $0.057 \pm 0.035$ & 0.087 & 0.125 \\
\hline Middle & $0.055 \pm 0.020$ & $0.052 \pm 0.018$ & $0.093 \pm 0.056$ & $0.061 \pm 0.069$ & 0.082 & 0.086 \\
\hline Mid-lower & $0.049 \pm 0.013$ & $0.058 \pm 0.018$ & $0.115 \pm 0.075$ & $0.163 \pm 0.101$ & 0.083 & 0.110 \\
\hline Lower & $0.066 \pm 0.026$ & $0.069 \pm 0.023$ & $0.144 \pm 0.076$ & $0.101 \pm 0.062$ & 0.101 & 0.131 \\
\hline
\end{tabular}

